\title{
REFAKENCE SYSTEMS AND FRAMES AS PROPOSED BY THE WORKING GROUP ON REFERENCE SYSTEMS
}

\author{
J. Kovalevsky \\ Chairman of the subgroup on coordinate frames and origins \\ Observatoire de la Côte d'Azur - CERGA \\ URA 1360 - Avenue Copernic - F- Grasse
}

\section{Introductory remarks}

Among the nine recommendations of the Working Group on Reference Frames as finalized during the Virginia Beach meeting, four are directly addressing the reference systems. They are the result of the work of the subgroup on coordinate frames and origins and other contributors whose names are given in section 6. The resulting document (Kovalevsky, 1991) was further revised by the working group as a whole and recommendations published (1991).

Kecommendation $n^{\circ} 1$ is the most fundamental one since it introduces General Relativity in the definition of celestial coordinate systems and sets the form of the metric to be used. This recommenda tion is presented by B. Guinot (1992) in this joint discussion, so I shall not comment upon it except to remark that it does not specify how the reference system has to be defined and how the reference frame realizing it is to be constructed. This is done by recommendations 2,6 and 7 that are explained in this presentation.

\section{General description of celestial reference frames}

Two fundamental considerations justify recommendation 2 . The first is that there must be several re ference frames in the solar system. Two are perequisite: a geocentric and a barycentric, the latter being centered at the barycentre of the solar system. Several others may be the useful in the future or even now is some particular studies (planetocentric, selenocentric, satellitocentric). The second point is also quite evident: the choice of a reference system should be such that its realization as a reference frame is the most accurate possible with the present observational capabilities.

This choice has been discussed at length in the subgroup and is among the following two possibilities :

i) To define the system by the dynamics of the solar system, as it was done in earlier days.

ii) To define the system by kinematic properties of some celestial bodies.

It is to remarked that in General Relativity, contrarily to the Newtonian case, they are not equiva lent. This can be summarized by the following statements : 
i) If a dynamical system is used, rotation, called geodetic precession, has to be applied in transformations between system centered at different points.

ii) If a kinematic system is used, there is no such rotation, but Coriolis terms must be introduced in the equations of motion written in any such system.

There is a certain symmetry in these additional complications so that they cannot serve as an argument in favour of one or the other definition. The working group has based its choice for a kinematic definition for a number of reasons, the principal being the following :

- While the position of planets is known with a very great accuracy, the extension of this accuracy to stars is far from being so precise.

- Experience of the construction of the FK5 shows that basing a fundamental system only on dynamical considerations is not accurate so that FK5 is partly a kinematic system based upon a model of galactic rotation.

- A large number of parameters are needed to define a dynamical system based upon the motions of planets: for instance, orbital elements at origin and planetary masses.

- VLBI is a technique with which one has already endeavoured to construct reference frames to a few tenths of a millisecond of arc, while the underlying theory is simply that, to a level of precision 10 or even 100 times better than the present observations, the Universe does not rotate and therefore remote extragalactic objects do not present a global rotation (note 2 of the recommenda tion).

The main drawback of this choice, namely that it is a radio frame not directly accessible in visible light is dealt with in recommendation 7 (section 4 of this paper).

The conclusion is that the kinematic system is the most accurate, spans the whole sky and is much less parameter dependent. This choice is stated in the first part of the recommendation. The se cond part of the recommendation concerns time scales and is not commented upon in this presentation. The third part presents a constraint on units which has a great impact on dynamical studies and on astronomical constants (note 5). Its consequences, however, do not affect the celestial reference frame directions that is to say classical astrometry. Other consequences are discussed in the presentation by B. Guinot (these Highlights).

Finally, the problem mentionned in connection with the choice of the type of reference system is dealt with in notes 3 and 4 where the implication that the geodetic precession (which is time variable since the relative position of the centres of coordinates is not fixed) has to be applied by introducing the corresponding Coriolis forces in the equations of motion.

\section{Realization of the kinematic reference system}

Recommendation 6 essentially states that the next step in the construction of the reference frame based upon distant extragalactic objects is to choose an as large as possible number of such objects to become primary fiducial points. This should be done by a working group defined in the recommendation that includes users of reference frames, people who observe and compile catalogues of positions of extragalactic radio sources and IERS where such frames have tentatively be constructed and used. The group should finalize its proposal in 1994. The intercomparison of existing or future catalogues provides necessary information for identifying the best sources and is therefore highly encouraged by the recommendation. 


\section{Observability and coordinate axis conditions}

Recommendation 7 has two distinct objectives that are stated in the two considerations. Let us examine them, starting with the second.

As mentionned in section 2, there is a problem with the choics at a reference system realized by radio observations: astronomers need to have a reference frame accessible to all types of observations, in all wavelengths among which visual observations play a major role.

Does this mean that we shall experience the same difficulty as mentionned in the case of a dynamical system, namely that the extension to star positions is not possible with a sufficient accuracy? The answer is that there exists ways of doing this transfer accurately (Argue, 1991). In comparison with a link to planets, link to extragalactic radio sources has the advantage that there will be much more reference sources than planets and that they will be uniformly distributed throughout the sky.

Radio stars are observed by VLBI as well as by HIPPARCOS. The precision already reached for a couple of radio stars observed during 7 years by VLBI is of the order of 0.15 milliseconds of arc for its five astrometric parameters (position, yearly proper motion and parallax). In addition, proper motions of some stars with respect to quasars are determined from old plates and new observations and a precision of 2 mas per year in the proper motion is currently reported. Finally, the possibility to use Hubble Space Telescope for this objective is not yet fully ruled out.

These observations will provide a link of the HIPPARCOS reference frame to theextragalactic with an accuracy of a few tenths of a millisecond of arc sometimes in 1996-97. This is just in time for the construction of the celestial reference frame defined by the present recommendations. If this will not be yet the case, note 5 states that FK5 will be provisionnally considered as the optical realization of the extragalactic reference system.

The construction of this link is the objective of 4-th part of the recommendation. However, it is more general. It recognizes that a dynamical system will continue to be used for some time forsolar system dynamical studies. Therefore, parts 3 and 4 call for a great effort of intercomparisons between existing reference frames of all types, including FK5.

Part 1 of the recommendation 7 does not strictly impose the axes of the new reference frame.Actually this is impossible at present, since results of intercomparisons are necessary to do it. But it sets an objective: to avoid as much as possible that the new coordinate system differs from the FK5 coordinate system at epoch J.2000.0 so that no discontinuity is introduced in proper motions and positions. Although this will not be strictly possible, it is expected that it should be smoother than the passage from FK4 to FK5. It would be desirable to use this opportunity to shift to astrometric parameters given by HIPPARCOS for the fundamental stars selected, so that the two discontinuities are introduces simultaneously.

Part 2 of the recommendation indicates how one should proceed in order to satisfy the condition on the axes set up in part 1 . It states that one should use the actually observed precession and nuta tion and not the conventional IAU series which are less accurate and would introduce spurious proper motions to otherwise fixed extragalactic objects. This is further emphasized by note 2 , while note 3 comments upon the way that the equinox at J.2000.0 should be set.

Note 4 draws the consequence of the absence of motion of the reference frame soconstructed. The origin and axes will remain fixed, so that in the future, all reference to ecliptic or equator and hence equinox will disappear. The new reference frame will become truly independent of Earth's motions. 


\section{Departure point for Earth rotation reckoning}

Departure point is the name recommended by the working group for what is known as "non-rotating origin". A proposal on the origin for reckoning the Earth rotation was sought, but members of the subgroup appeared not to be prepared for such decision and very contradictory civinions were expressed on this matter. A number of studies have been made on the use of the departure point in Earth rotation studies and in astrometry. Some decision should, however, be taken at the next General Assembly. This is because the equinox will no more be fit for this purpose once the extragalactic reference frame will come to existence since its position will be totally independent from the reference system. Evidently, the origin of the Earth rotation angle should be on the equator, but in definition must some how involue the origin of the reference system. Several possibilities have been proposed, one of which is the intersection $\mathrm{H}$ of the moving medirian plane of the origin of the celestial reference frame with the moving equator. Another is the departure point $\varepsilon$ which is very close to $\mathrm{H}$ and has the advantage to override the use of a conventional precession-nutation series (Capitaine and Gontier, 1991) and to give the true rate of rotation at any instant.

\section{Contributors to the work of the subgroup}

The contribution of the following people is acknowledged.

- Members of the subgroup on coordinate frames and origins : V.K. Abalakin, S. Aoki, F. Arias, C. Boucher, N. Capitaine, K. Johnson, J. Kovalevsky, C. Ma, I.I. Mueller, C.A. Murray, H. Schwan, C.A. Smith, C. de Vegt and R. Wielen.

- Other contributors to the work of the subgroup : V.A. Brumberg, T. Damour, J. Dickey, M. Feissel, T. Fukushima, B. Guinot, T. Huang, M. Standish, J.G. Williams and B.X. Xu.

\section{References}

Argue, N., 1991, "Hipparcos: its link to extragalactic reference frame", in Reference systems, IAU Coll. 127, J.A. Hughes, C.A. Smith and G.H. Kaplan (eds.), US Naval Observatory Publ., p. 63.

Capitaine, N. and Gontier, A.M., 1991, "Procedures for VLBI estimates of the Earth rotationparameters using the non-rotating origin", in Reference systems, IAU Coll. 127, J.A. Hughes, C.A. Smith and G.H. Kaplan (eds.), US Naval Observatory Publ., p. 77.

Guinot, B., 1992, this volume.

Kovalevsky, J., 1991, "Preliminary report of the work of the subgroup coordinate frames and origins", in Reference systems, IAU Coll. 127, J.A. Hughes, C.A. Smith and G.H. Kaplan (eds.), US Naval Observatory Publ., p. 17.

Recommendations of the working group on reference systems, in Reference systems, IAU Coll. 127, J.A. Hughes, C.A. Smith and G.H. Kaplan (eds.), US Naval Observatory Publ., p. 408. 